\title{
Immunohistochemical evaluation of induced Kidney injury using UUO model
}

\author{
F.Z. Mohammed ${ }^{1}$, I.M. EL-Deen ${ }^{2}$, S.M. Hamed ${ }^{3}$ and N.N. EL-Sonbaty ${ }^{* 1}$ \\ ${ }^{1}$ Department of Chemistry, Faculty of science, Zagazig University, Egypt. \\ ${ }^{2}$ Department of Chemistry, Faculty of science, Port Said University, Egypt. \\ 3 Urology and Nephrology Center, Mansoura University, Egypt.
}

Received: 15 May 2016/ Accepted: 11 July 2016

*Corresponding author: dr.nanes@gmail.com

\begin{abstract}
Fibrosis is a major factor in the progressive loss of renal function in patients with a wide variety of kidney diseases. Interstitial fibrosis has a major role in the progression of renal diseases. It is widely recognized that progressive renal disease is accompanied by tubulointerstitial changes characterized by tubular atrophy, increased number of interstitial fibroblasts, phenotypic change of interstitial cells, accumulation of matrix proteins, and interstitial infiltrate of mononuclear cells. Several animal models are available for the study of renal fibrosis. A model of renal fibrosis that encompasses many aspects of other models of kidney disease is unilateral ureteral obstruction (UUO). UUO has emerged as an important model for the study of the mechanisms of renal fibrosis and also for the evaluation of the impact of potential therapeutic approaches to renal disease. Many quantifiable pathophysiological events occur over the span of $1 \mathrm{wk}$ of UUO, making this an attractive model for study. There are many readily quantifiable cellular and molecular events during the initiation and progression of renal fibrosis that make UUO an increasingly good experimental model for study.

Keywords: immunohistochemical stain, kidney injury, renal fibrosis, TNF, unilateral ureteral obstruction (UUO).
\end{abstract}

\section{Introduction}

The most severe forms of kidney disease are acute kidney injury (AKI) and chronic kidney disease (CKD). CKD is divided into five stages, according to estimated GFR (Glomerular filtration rate) [1]. Acute kidney injury (AKI) is the syndrome arising from a rapid fall in GFR. It is characterized by retention of both nitrogenous and non- nitrogenous waste products of metabolism, as well as disordered electrolyte, acid-base and fluid homeostasis. There is evidence that even relatively small acute reductions in kidney function are associated with poorer outcomes, including increase mortality and higher risk of long-term dialysis [2]. AKI categorize to Pre-renal AKI, Intrinsic renal AKI, and Post-renal AKI [3]. Recovery of renal function will 
depend on underlying diagnosis. For ATN (Acute tubular necrosis), 50\% will have some degree of residual renal impairment. This will be irreversible, dialysisdependent renal failure in $75 \%$ (10\% in the elderly). The risk of worsening or de novo CKD and death following an episode of AKI (even if function appears to return to normal) is high [3]. A number of terms have been applied to the pathological process of renal accumulation of collagen or extracellular matrix: fibrosis, sclerosis, and scarring. It is widely recognized that progressive renal disease is accompanied by tubulointerstitial changes characterized by tubular atrophy, increased number of interstitial fibroblasts, phenotypic change of interstitial cells, accumulation of matrix proteins, and interstitial infiltrate of mononuclear cells [4]. A model of renal fibrosis that encompasses many aspects of other models of kidney disease is unilateral ureteral obstruction (UUO) The model of UUO in the rodent generates progressive renal disease. UUO has emerged as an important model for the study of the mechanisms of renal fibrosis. Surgically created UUO can be experimentally manipulated with respect to timing, severity, and duration. Unilateral ureteral obstruction results in marked renal hemodynamic and metabolic changes, followed by tubular injury and cell death by apoptosis or necrosis, with interstitial macrophage infiltration. Proliferation of interstitial fibroblasts with myofibroblast transformation leads to excess deposition of the extracellular matrix and renal fibrosis [4]. If the insult is removed (by relief of obstruction) renal injury may be prevented or reversed [5]. Management principles for acute UUO are to rule out infection, then identify and relieve the obstructing lesion. UUO induces after a few hours cellular infiltration in the tubulointerstitium [6]. Most of the evidence suggests that the rodent model of UUO is reflective of human renal disease processes [7].

The advantages of using UUO as a model of renal fibrosis include the absence of an exogenous toxin, the lack of a 'uremic' environment, and the availability of the contra lateral kidney as a control. Using the contra lateral kidney as a control, however, does not take into account its cellular, metabolic and functional renal compensatory changes in response to UUO. Surgical models of UUO have the added advantage of allowing variation in the severity, timing, and duration of obstruction, as well as the opportunity to study recovery following relief of the obstruction [8].

Complete UUO initiates a rapid sequence of events in the obstructed kidney, leading within $24 \mathrm{~h}$ to reduce renal blood flow and glomerular filtration rate. This is followed within several days by hydronephrosis, interstitial inflammatory infiltration (macrophages) and tubular cell death attribute able to apoptosis and necrosis. Tubular epithelial cell death is caused by a number of stressors resulting from UUO, including ischemia, hypoxia, oxidant injury, and axial strain caused by tubular dilatation. There appears to be a close association between progressive fibrosis and tubular cell death [9]. Following complete UUO in the rat or mouse, the progression to a severely hydronephrotic kidney with marked loss of renal parenchyma takes place over 1-2 weeks, with more severe fibrosis in the neonate than the adult. Because most cases of clinical congenital obstructive nephropathy involve partial, rather than complete obstruction, models of partial UUO have been developed in the neonatal rat and mouse. These models are also useful in the study of the pathogenesis of renal fibrosis, because the lesions develop more slowly and may be better suited to the study of therapeutic manipulations [10]. In addition to the classical mechanical view of UUO, there is evidence that the pathophysiological process of nephron destruction, although initiated by an increased intratubular hydrostatic pressure and secondary ischemia, is later magnified by cellular processes that can be classified into three broad categories: tubulointerstitial inflammation, tubular cell death and fibrosis [11].

\section{A. Interstitial Inflammation}

In the UUO model, interstitial infiltration by macrophages progressively increases from 12 hours after obstruction to up to 14 
days. UUO shares mediators of inflammation with other forms of renal injury and with tissue injury in general. AngII has a central role in initiation and progression of obstructive nephropathy, both directly and indirectly, by stimulating production of molecules that contribute to renal injury. AngII and tumor necrosis factor-alpha $(\mathrm{TNF} \alpha)$ are clearly upregulated in this model and together with other mediators activate NF- $\mathrm{KB}$ [11].

\section{B. Tubular apoptosis}

In UUO, tubular atrophy is the result of an increased rate of tubular cell apoptosis, with the contribution of other processes such as epithelial-mesenchymal transition (EMT) [12]. Most apoptosis in UUO takes place in tubular cells, but also interstitial cells undergo apoptosis. The latter may contribute to resolution of inflammation and repair of fibrosis [13].

\section{Tubulointerstitial fibrosis}

Progressive interstitial fibrosis is the most dramatic consequence of chronic obstructive nephropathy, and is the final common pathway of all forms of CKD. Long-term activation of interstitial fibroblasts results in proliferation and excessive ECM accumulation [14].The main known regulators of interstitial fibrosis in UUO are AngII, connective tissue growth factor (CTGF), bone morphogenetic protein-7 (BMP-7), and the plasminogen-plasmin axis $[15,16]$.

\section{Role of TNF in Kidney Injury}

Members of the TNF super family participate in kidney disease. Tumor necrosis factor (TNF) and Fas ligand regulate renal cell survival and inflammation and therapeutic targeting improves the outcome of experimental renal injury. TNF-related apoptosisinducing ligand (TRAIL) and its potential decoy receptor osteoprotegerin are the two most upregulated death-related genes in human nephropathy [17]. TNF (TNFSF2) was the first member of the family to be implicated in the pathogenesis of kidney injury. TNF also has an immunoregulatory role. In the kidney, TNF is expressed, synthesized, and released by infiltrating macrophages and by intrinsic kidney cells, namely, endothelial, mesangial, glomerular, and tubular epithelial cells. In vivo, the TNF expression pattern seems to be related to the primary kidney injured [18]. TNF activates two receptors, TNFR1 and TNFR2. TNFR1 is present in normal glomeruli and is upregulated on infiltrating leukocytes in response to renal injury. TNFR2 is usually not expressed in normal kidney and is upregulated in tubular cells in response to renal injury [19]. Increasing evidence has implicated TNF as a major participant in the pathogenesis of kidney injury, promoting inflammation, apoptosis, and accumulation of extracellular matrix, reducing glomerular blood flow and damaging the glomerular permeability barrier with development of albuminuria [20].Thus TNF- $\alpha$ may have a role in initiating tubulointerstitial injury in the obstructed kidney [18].

\section{Material and Method}

\section{Experimental Animal}

Healthy C57BL/6 mice were obtained from serum and vaccine authority in Cairo. Their ages were ranged from 8- 10 weeks and their weights were in the range of 20- 23 grams. On arrival, the animals were placed at random and allocated to groups in polypropylene cages with paddy husk as bedding. Animals were housed at a temperature of $24^{\circ} \mathrm{C}$ and relative humidity of 30-70\%. A 12:12 h, light: dark cycle was followed. They had free access to standard mice food and water.

\section{Experimental design (protocol)}

The animals were randomly divided into four groups. With the mice under pentobarbital anesthesia, the left ureter were ligated with 4-0 silk at two locations and cut between the ligatures to prevent retrograde urinary tract infection at the ureteropelvic junction

The first group (Sham) 
Consist of 5 mice that operation performed had their ureters manipulated but not ligated.

\section{The second group}

Consist of 10 mice that were sacrificed at 3 days after uuo operation.

\section{The third group}

Consist of 10 mice that were sacrificed at 7 days after uuo operation.

\section{The fourth group}

Consist of 10 mice that were sacrificed at 14 days after uuo operation.

At the end of experiment, operated mice were killed under pentobarbital anesthesia 3, 7 and 14 days after UUO. Blood was collected. Kidneys were removed, cut transversely into 3 slices. One part was embed in Tissue Tek (OCT compound) for immunofluorescent study, the second part was snap-frozen in liquid nitrogen for genetic study and the last slice was fixed in $10 \%$ buffered formalin, and embedded in paraffin for morphological studies.

\section{Biochemical Evaluation of Interstitial Fibrosis}

Creatinine and BUN were measured in all mice involved in this study.

Table 1 Effect of uuo on BUN and Creatinine

\begin{tabular}{c|c|c|c|c|}
\hline Parameters & Control & 3 Days & 7 Days & 14 Days \\
\hline Groups & & & & \\
\hline Bun M \pm SD & $47.053 \pm 0.04$ & $0.51^{* * *} \pm 0.15$ & $0.36^{* * *} \pm 0.05$ & $0.46^{* * *} \pm 0.05$ \\
\hline
\end{tabular}

$\mathrm{SD}=$ Standard deviation, $\mathrm{M}=$ Mean, $\mathrm{BUN}=$ Blood urea nitrogen, $*=\mathrm{P}<0.05, * *=\mathrm{P}<0.01, * * *=\mathrm{P}<0.001$.

Effect of uио on Morphological charachteristic of kidney:

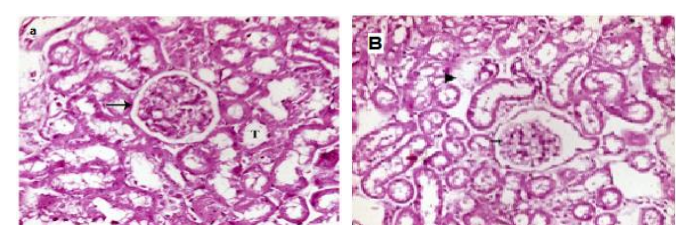

Fig 1 Section of mice kidney that sacrificed at 3 days after uuo. A(Sham); kidney showed normal renal corpuscle (arrow) and renal tubules $(\mathrm{T})$ lined by cuboidal to columnar epithelium (HE, $400 \mathrm{X}$ ). B; kidney showed dilation of renal glomeruli (arrow) and loss of renal tubules (arrow head) (HE, $400 \mathrm{X}$ ).

\section{Morphometric Evaluation of Interstitial Fibrosis}

Routine HE stain was stained for $4 \mu \mathrm{m}$ sections of the kidney. The results were expressed as percentage of the measured area, which represented the injury.

\section{Immunohistochemical Studies}

Indirect immunohistochemical stain will be performed to detect type III, type IV collagen and TNF as markers for tubulointerstitial injury.

\section{Results}

\section{Effect of uиo on biochemical parameters in blood}

Results showed a significant decrease in creatinine concentration in mice sacrificed at 3 days, 7 days and 14 days after uuo operation $52 \%, 66 \%$ and $57 \%$ respectively compared to control group. Results showed a significant increase in BUN concentration in mice sacrificed at 3 days, 7 days and 14 days after uuo operation $17 \%, 42 \%$ and $66 \%$ respectively compared to control group (Table 1). 


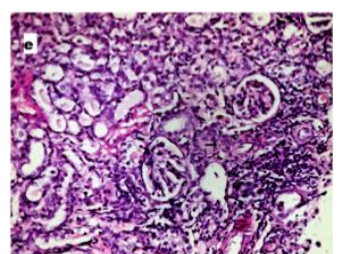

Fig 3 Section of kidney of mice sacrificed at 14 days after uuo. E; kidney showed necrosis of renal tubules and glomeruli, an extensive replacement of renal tissue with chronic inflammatory exudates with fibroblastic proliferation (arrow) (HE, $400 \mathrm{X}$ ).

The effect of uuo on TNF in kidney tissue using Immunohistochemical stain
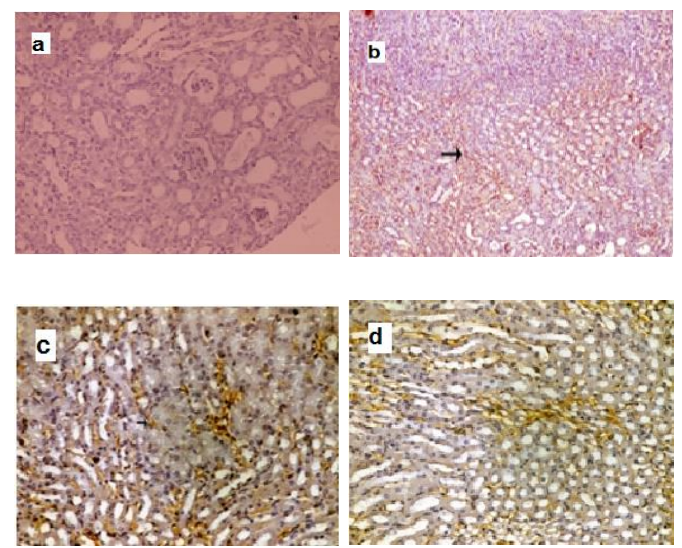

Fig 4 Section of kidney of mice sacrificed at3, 7 and 14 days after uuo. A; Sham, kidney showed negative for TNF immunostain (400 X). b; at 3 days after uuo, kidney showed a moderate brown stained renal tubular epithelium (arrow) (100 X). c; At 7days after uuo, kidney showed a brown color in interstitial tissue (arrow) (400 X). d; at 14days after uuo, extensive brown color in renal tubular epithelium and interstitial tissue (400 X).

The effect of uиo on collagen in kidney tissue using Immunohistochemical stain:
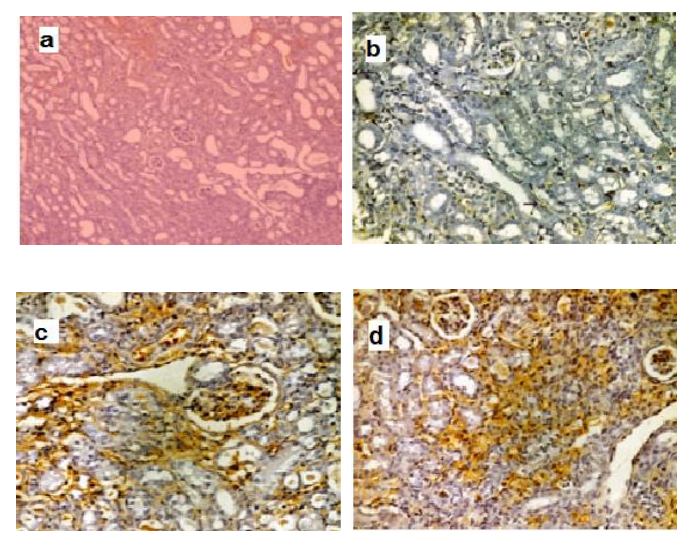

Fig 5 Section of kidney of mice sacrificed at 3, 7 and 14 days after uuo. a; Sham showed negative stained for collagen $(100 \mathrm{X})$. b; At 3days after uuo, kidney showed a mild brownish stained collagen in interstitial tissue (arrow) (400 X). c; At 7days after uuo, the renal glomeruli (arrow) and interstitial tissue exhibit brown stain for collagen that confirming renal sclerosis $(400 \mathrm{X})$. d; At 14days after uuo, kidney showed an intense brownish stained collagen in the renal tissue $(400 \mathrm{X})$.

\section{Discussion}

The work with this model showed biochemically the pathogenic mechanisms of induced tubulointerstitial injury by UUO in kidney using mouse as an animal model. The results of the present work study different parameters where this model combines several key advantages for studying development and progression of kidney injury, including assessment of functional consequences of kidney injury using biochemical evaluation in blood such as blood urea nitrogen (BUN), serum creatinin measurements and the ability to study pathophysiology during kidney injury through morphometric evaluation of interstitial fibrosis within groups at different unilateral ureteral obstruction (UUO) sacrifice time 3, 7 and 14 days to show the developments during kidney injury in glomular infiltration, interstitial inflammation, interstitial fibrosis, necrosis and dilated tubules by using hematoxylene and Eiosn $(\mathrm{H} \& \mathrm{E})$ stain, special stain as MT representing fibrosis and PAS representing.In this study, biochemically investigations measured to monitor changes due to UUO we found that; creatinine shows a significant decrease comparing control group within 3 and 7 days then raise at day 14 but still less than sham creatinine level. These findings were in contrary with Ma et al. [21], where they found no significant differences in different time groups. BUN level in this study, showed a significant increase within 3, 7 days and this increase was not statistically detected $(\mathrm{P}<0.49$ and 0.24 respectively). At 14 days this increase was statistically significant, $\mathrm{P}<0.001$. While these findings were in agreement with Puri et al. [22] they found also significant increase after 6 days on. Histopathological examination of different markers were done to monitor the development of kidney injury specially fibrosis. Using hematoxylene and Eiosn $(\mathrm{H}$ 
\& E) stain at different UUO groups, we found no significant histological abnormality in control groups while kidney shows morphomatically changes as dilation of renal glomeruli, dilatation of renal tubules that appeared from day 3 and loss of renal tubules with degeneration of the renal parenchyma at day 7 which become more sever at day 14 and fibrotic tissue replace almost all the kidney structure. These findings were in compatible with Chung et al. [23], where they found tubular injury in the obstructed kidney which markedly increased after UUO. TNF was implicated in the pathogenesis of kidney injury. It is a potent pro-inflammatory cytokine and an important mediator of inflammatory tissue damage. TNF also has an immunoregulatory role Mas et al. [24]. By using Immunohistochemical stain monitoring TNF severity at different UUO sacrifice time. The intensities of TNF stain were increased gradually within different UUO groups confirming renal injury. Collagen also monitored by immunostaining is representing renal damage. It also shows gradually increase in collagen intensities in dependent manner with different UUO sacrifice time leading to a complete structures and function loss. These findings were in compatible with Sun et al. [25], where they found the severity of renal collagen also increased after UUO in a time-dependent manner.

\section{Conclusion}

In conclusion, this work showed that C57BL/6 mice can be taken as a good model for studying the mechanism of UUO changes while typical biochemical morphological changes have been provided through different parameters measured in each item. With this model we may trying a treatment concerning the different changes we have been found during the study due to establishment of a valid mouse model of kidney diseases (KD) facilitate testing of diagnostic and therapeutic interventions in mice before testing in human.

\section{References}

1. Simon Steddon, Neil Ashman, Alistair Chesser, John Cunningham (2014): Chronic kidney disease, oxford medical publications oxford handbook of nephrology and hypertension; (3), 192-197

2. Ricci Z, Ronco C, D Amico G, et al. (2006) Practice patterns in the management of acute renal failure in the critically ill patient: an international survey. Nephrology Dialysis Transplantation; 21, 690696.

3. Bucaloiu ID, Kirchner HL, Norfolk ER, et al. (2012) Increased risk of death and de novo chronic kidney disease following reversible acute kidney injury. Kidney International; 81: $477-485$.

4. Robert L. Chevalier1, Michael S. Forbes1 and Barbara A, Thornhill (2009) Ureteral obstruction as a model of renal interstitial fibrosis and obstructive nephropathy. Kidney International; 75, 1145-1152

5. Schnaper HW, Kopp JB (2006) Why kidneys fail: Report from an American Society of Nephrology Advances in Research Conference. J Am Soc Nephol; 17: 1777-1781.

6. Bascands,JL and Schanstra, JP (2005) Obstructive nephropathy: insights from genetically engineered animals. Kidney Int.; 68(3): 925-937.

7. Klahr S \& Morrissey J (2002): Obstructive nephropathy and renal fibrosis. Am J Physiol Renal Physiol; 283: F861- F75.

8. Docherty NG, O'Sullivan OE, Healy DA et al (2006) Evidence that inhibition of tubular cell apoptosis protects against renal damage and development of fibrosis following ureteric obstruction. Am J Physiol Renal Physiol; 290: F4-F13, Jan.F4$13, / 1$.

9. Thornhill BA, Burt LA, Chen $\mathrm{C}$ et al (2007) Variable chronic partial ureteral obstruction in the neonatal rat: A new model of ureteropelvic junction obstruction. Kidney Int; 67: 42-52.

10. Chen CO, Park MH, Forbes MS et al (2007) Angiotensin converting enzyme inhibition aggravates renal interstitial injury resulting from partial unilateral ureteral obstruction in the 
neonatal rat. Am J Physiol Renal Physiol; 292: F946-955.

11. Yoo K, Thornhill B, Forbes M, et al (2006) Osteopontin regulates renal apoptosis and interstitial fibrosis in neonatal chronic unilateral ureteral obstruction. Kidney Int; 70(10):17351741

12. Sanz A, Santamara B, Ruiz-Ortega M, Egido J, Ortiz A (2008) Mechanisms of renal apoptosis in health and disease. J Am Soc Nephrol; 19(9):1634- 1642.

13. Misaki T, Yamamoto T, Suzuki S, et al (2009) Decrease in tumor necrosis factor-alpha receptor-associated death domain results from ubiquity independent degradation in obstructive renal injury in rats. Am J Pathol; 175(1):74- 83.

14. Strutz F, Zeisberg M (2006) Renal fibroblasts and myofibroblasts in chronic kidney disease. J Am Soc Nephrol; 17(11):2992-2998.

15. Lange-Sperandio B, Trautmann A, Eickelberg O et al (2007) Leukocytes induce epithelial to mesenchymal transition after unilateral ureteral obstruction in neonatal mice. Am J Pathol; 171(3):861-871.

16. Lin S, Kisseleva T, Brenner D, Duffield J (2008) Pericytes and perivascular fibroblasts are the primary source of collagen-producing cells in obstructive fibrosis of the kidney. Am J Pathol; 173(6):16171627.

17. Maria D. Sanchez-Ni, Alberto BenitoMartin, Sara Gonc alves, Ana B. Sanz, Alvaro C. Ucero, Maria C. Izquierdo, AdrianM. Ramos, Sergio Berzal, Rafael Selgas, Marta Ruiz-Ortega, Jesus Egido and Alberto Ortiz1 (2010) TNF Superfamily: A Growing Saga of Kidney Injury Modulators. Hindawi Publishing Corporation, Mediators of Inflammation; 182958, 11 pages doi:10.1155/2010/182958

18. Ernandez T. and Mayadas T. N. (2009) "Immunoregulatory role of TNF $\alpha$ in inflammatory kidney diseases,"
Kidney International; vol. 76, no. 3, pp. 262-276.

19. Tansey M. G. and Szymkowski D. E. (2009) The TNF superfamily, new pathways, new indications and new drugs," Drug Discovery Today; vol. 14, no. 23-24, pp. 1082-1088

20. Khan S. B, Cook H. T, Bhangal G, Smith J, K.Tam F. W, and Pusey C. D (2005) "Antibody blockade of TNF- $\alpha$ reduces inflammation and scarring in experimental crescentic glomerulonephritis," Kidney International; vol. 67, no. 5,pp. 18121820.

21. Ma Y.Y, Sun D, Li J, and Yin Z.C (2010) Department of Nephrology, Affiliated Hospital of Xuzhou Medical College, Xuzhou, PR China," Life sciences, 86, 21-22, 798-807. DOI: 10.1016/j.lfs.2010.03.013.

22. Puri T. S, Shakaib M. I, Chang A et al (2010) "Chronic kidney disease induced in mice by reversible unilateral ureteral obstruction is dependent on genetic background," American Journal of Physiology: Renal Physiology, vol. 298, no. 4, pp. F1024-F1032.

23. Chung S, Yoon H.E, S.J. Kim, Kim S.J, Koh E.S, Y.A. Hong, Park C.W, Chang Y.S, and Shin S.J (2014) "Oleanolic acid attenuates renal fibrosis in mice with unilateral ureteral obstruction via facilitating nuclear translocation of Nrf2," Nutrition \& Metabolism,

24. Mas S, Mart'ınez-Pinna R, Mart'inVentura J. L et al (2010) "Local nonesterified fatty acids correlate with inflammation in atheroma plaques of patients with type 2 diabetes," Diabetes; vol. 59, no. 6, pp. 12921301.

25. Sun YBY, Qu X, Li $X$, NikolicPaterson DJ, Li J (2013): Endothelial Dysfunction Exacerbates Renal Interstitial Fibrosis through Enhancing Fibroblast Smad3 Linker Phosphorylation in the Mouse Obstructed Kidney. PLoS ONE, 8(12). 


\section{الملخص العربي}

عنوان البحث: تقييم مناعى لاصابة الكلى باستخدام انسداد أحادي الحالب كنموذج

فاتن زهران1، ابراهيم محى الدين²، سحر حامد3، نانيس السنباطى2

1 قسم الكيمياء ـ كلية الطوم - جامعة الزقازيق

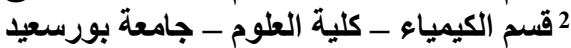

3 مركز الكلى والمسالك - جامعة المنصورة

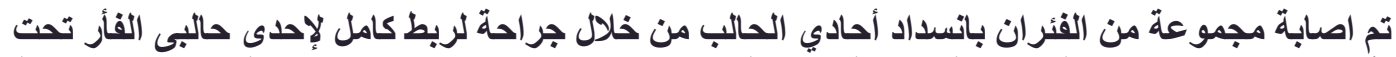

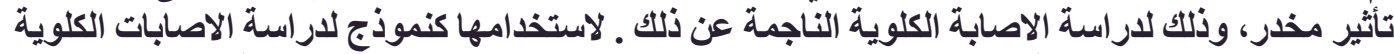

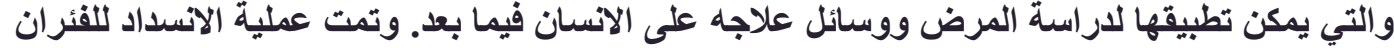

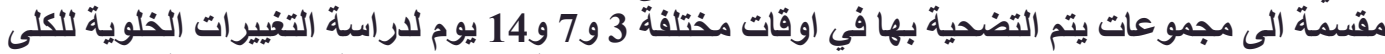
خلال تلكك الفترات. واوضحت النتائج حدوث تغييرات مورفئ موفولوجية للخلايا الكلوية ادت باتلنهاية إلى فقدانها

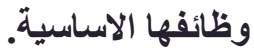

